\title{
Integrated bioinformatics analysis for the identification of key genes and signaling pathways in thyroid carcinoma
}

\author{
BO ZHANG ${ }^{1,2^{*}}$, ZUOYU CHEN $^{1 *}$, YUYUN WANG $^{1}$, GUIDONG FAN $^{2}$ and XIANGHUI HE ${ }^{1}$ \\ ${ }^{1}$ Department of General Surgery, Tianjin Medical University General Hospital, Tianjin 300052; \\ ${ }^{2}$ Department of General Surgery, Inner Mongolia Autonomous Region People's Hospital, \\ Hohhot, Inner Mongolia 010017, P.R. China
}

Received May 30, 2020; Accepted December 1, 2020

DOI: $10.3892 /$ etm.2021.9729

\begin{abstract}
Thyroid carcinoma (TC) is one of the most common types of endocrine neoplasm with poor prognosis due to its aggressive behavior. Biomarkers for early diagnosis and prevention of TC are in urgent demand. By using a bioinformatics analysis, the present study aimed to identify essential genes and pathways associated with TC. First, the GSE27155 and GSE50901 expression profiles were downloaded from the Gene Expression Omnibus database. Differentially expressed genes (DEGs) were obtained using the two microarray datasets and further subjected to integrated analysis. A gene ontology (GO) and Kyoto Encyclopedia of Genes and Genomes (KEGG) pathway enrichment analysis revealed 45 common DEGs in the two datasets. GO and KEGG pathway analysis indicated that the biological functions of the DEGs included protein binding, cardiac muscle cell potential involved in contraction, aldehyde dehydrogenase activity, the TGF- $\beta$ receptor signaling pathway and the canonical Wnt signaling pathway. A protein-protein interaction network was also constructed and visualized to display the nodes of the top 9 up- and 36 downregulated common DEGs. The integrated bioinformatics analysis indicated that potassium inwardly rectifying channel subfamily $\mathbf{J}$ member 2 (KCNJ2) was the most significantly upregulated DEG. The transcriptional levels of $\mathrm{KCNJ} 2$ were confirmed to be elevated in TC tissues compared with those in normal tissues using reverse transcription-quantitative PCR analysis. Furthermore, the expression level of KCNJ2 was significantly associated with the 5-year survival rate of patients with TC, which was determined using the Kaplan-Meier method. In TC cell lines, KCNJ2 was also
\end{abstract}

Correspondence to: Dr Xianghui He, Department of General Surgery, Tianjin Medical University General Hospital, 154 Anshan Road, Heping, Tianjin 300052, P.R. China

E-mail: hexh88@tmu.edu.cn

${ }^{*}$ Contributed equally

Key words: thyroid cancer, Gene Expression Omnibus, differentially expressed genes, integrated bioinformatics, KCNJ2 upregulated as compared with that in a normal control cell line. Finally, small interfering RNA was used to knock down the expression of $\mathrm{KCNJ} 2$, which was demonstrated to inhibit cell proliferation, migration and invasion, while increasing apoptosis in TC cells. In conclusion, in the present study, $\mathrm{KCNJ} 2$ was screened as an oncogene with a crucial role in TC development and progression and may represent a promising candidate biomarker and therapeutic target for TC.

\section{Introduction}

As the most common endocrine malignancy, thyroid cancer (TC) has had an increasing incidence worldwide in recent years (1-3). TC currently accounts for $0.5-1 \%$ of all human cancers and ranks fifth in prevalence amongst cancers in females $(4,5)$. TC may be classified into several subtypes based on its histopathological characteristics, such as papillary thyroid carcinoma (PTC) and anaplastic thyroid carcinoma (ATC) (6-9). Due to the aggressive behavior of $\mathrm{TC}$, the 5-year survival rate of patients with $\mathrm{TC}$ is poor $(7,10)$. Hence, initial diagnosis at an earlier stage may be beneficial for improving the poor 5-year survival rate of patients with TC. In this light, it is required to discover promising diagnostic and prognostic biomarkers for TC.

Microarray technology is a highly efficient technique, which has been widely used for gene-expression profiling (11). In the past decade, numerous studies have used microarray technology to investigate the differentially expressed genes (DEGs) and determine distinct pathways in which they are accumulated, including those in the categories biological process (BP), molecular function (MF) and cellular component (CC), and to identify significant genes associated with tumorigenesis that may serve as potential biomarkers for the diagnosis or treatment of cancer (12-14). Owing to different backgrounds and technological detection platforms for the analysis of collected samples, the identification of DEGs in each independent experiment is not always the same. Thus, integrated bioinformatics analysis may be performed to enable a comprehensive analysis of changes in gene expression and provide an effective way to explore candidate biomarkers for various cancer types $(5,15,16)$. Previous studies have generated and analyzed gene expression profiles for TC and screened thousands of DEGs, which may be involved in the progression 
of TC $(5,8)$. However, there is currently no promising biomarker with diagnostic and prognostic applications in TC.

In the present study, the mRNA expression profiles of the datasets GSE27155 (17) and GSE50901 (18) were downloaded from the GeneExpression Omnibus (GEO) database to obtain the DEGs for integrated bioinformatics analysis. Gene ontology (GO) and Kyoto Encyclopedia of Genes and Genomes (KEGG) pathway enrichment analyses indicated that the biological functions of the DEGs were mainly focused on protein binding, cardiac muscle cell potential involved in contraction, aldehyde dehydrogenase activity, TGF- $\beta$ receptor signaling pathway and the canonical Wnt signaling pathway. Subsequently, a protein-protein interaction (PPI) network was drawn to display common interactions among the screened DEGs. Of note, potassium inwardly rectifying channel subfamily $\mathbf{J}$ member 2 (KCNJ2) was the most significantly and commonly upregulated among the DEGs and the upregulation of $\mathrm{KCNJ} 2$ was then confirmed in tissues and cell lines of TC. Furthermore, the expression level of KCNJ was determined to be significantly associated with the 5-year survival rate of patients with TC. The potential role of KCNJ2 to regulate the growth, survival and metastatic properties of TC cells was determined by using small interfering (si)RNA to knock down the expression of KCNJ2. The results suggested that the KCNJ2 gene may have a significant role during TC development and progression and serve as a promising diagnostic biomarker and therapeutic target for TC.

\section{Materials and methods}

Microarray data. GEO (https://www.ncbi.nlm.nih.gov/geo/) is a public repository genomics database, which may be queried for all data sets. The GSE27155 and GSE50901 gene expression profiles were downloaded from GEO. The datasets were selected based on the following criteria: i) TC tissue samples were used; ii) the adjacent normal tissues were used as a control; iii) information on the technology and platform was provided.

Identification of DEGs. The statistical analysis software $\mathrm{R}$ (version 3.5.0; https://www.r-project.org/) and Bioconductor packages (http://www.bioconductor.org/) were respectively used to evaluate the significance of differences in gene expression between TC tissues and the corresponding normal tissues in the two data sets. To generate more reliable results, normalization and base-2 logarithm conversion for the matrix data of each GEO dataset were performed using the limma package in $\mathrm{R}$ software. Differential expression analysis was then performed by comparing cancer tissues with control tissues. Genes with $\mid \log$ fold change (FC) $\mid>1, \mathrm{P}<0.05$ and adjusted $\mathrm{P}<0.05$ were considered significant DEGs.

Functional and pathway enrichment analysis. The Database for Annotation, Visualization and Integrated Discovery (DAVID) database (https://david.ncifcrf.gov/) is a useful website for high-throughput gene function analysis. Based on the DAVID database, functional and pathway enrichment of candidate genes and annotation were conducted. The DAVID online tool was used to perform the GO (http://www.geneontology.org/) annotations for the screened DEGs, defining gene functions and dividing them into 3 categories: MF, CC and BP. The KOBAS database (http:/kobas.cbi.pku.edu.cn/) was used for KEGG pathway analysis. In the study, the DEGs determined from integrated microarray data for TC were analyzed and statistical significance was set at $\mathrm{P}<0.05$.

PPI interaction network analysis. The PPI network integration of the screened DEGs was performed using the Search Tool for the Retrieval of Interacting Genes and proteins (STRING) online database (http://string-db.org/). Cytoscape (http://www. cytoscape.org/) was used to construct and visualize the PPI interaction network.

Clinical specimens. Between March 8, 2015 and March 8, 2018, TC and matched normal tissues were collected from 41 patients at the Inner Mongolia Autonomous Region People's Hospital (Hohhot, China). The survival data of the patients over 5 years were collected to determine the 5-year overall survival rate (with the time of survival defined as the time from prospective enrollment until death due to any reason) by using Kaplan-Meier method and estimate the prognosistic value of biomarkers. Prior to surgery, no systemic chemotherapy or radiotherapy treatment was performed on any of the patients with TC. Before the tissues were used, all patients provided written informed consent. The study was approved by the ethics committee of the Inner Mongolia Autonomous Region People's Hospital (Hohhot, China). All specimens were maintained at $-80^{\circ} \mathrm{C}$ until use.

Cell culture. The TC cell lines GLAG-66, CAL-62, B-CPAP, TPC-1 and SW1736 were authenticated by STR profiling and cultured in Dulbecco's modified Eagle's medium (Invitrogen; Thermo Fisher Scientific, Inc.) with 10\% heat-inactivated fetal bovine serum (FBS; Gibco; Thermo Fisher Scientific, Inc.). The human normal thyroid cell line Nthy-ori 3-1 was cultured in RPMI-1640 medium (Gibco; Thermo Fisher Scientific, Inc.) with $10 \%$ heat-inactivated FBS. All cultured cells were incubated in a humidified atmosphere containing $5 \% \mathrm{CO}_{2}$ at $37^{\circ} \mathrm{C}$. All cell lines were sourced from the Institute of Biochemistry and Cell Biology of the Chinese Academy of Science.

Reverse transcription-quantitative (RT-q)PCR. Total RNA was extracted from specimens and cultured cells with TRIzol@ reagent (Thermo Fisher Scientific, Inc.) following the manufacturer's protocol. Total RNA $(1 \mu \mathrm{g})$ was reverse transribed to cDNA using the HiScript First Strand cDNA Synthesis Kit (Containing Buffer, RNase Inhibitor, dNTP and Transcriptase; Vazyme Biotech Co., Ltd.). The temperature protocol was as follows: $25^{\circ} \mathrm{C}$ for $5 \mathrm{~min}, 37^{\circ} \mathrm{C}$ for $45 \mathrm{~min}$ and $85^{\circ} \mathrm{C}$ for $5 \mathrm{sec}$. qPCR was conducted in an ABI StepOnePlus ${ }^{\mathrm{TM}}$ real-time PCR system (Applied Biosystems; Thermo Fisher Scientific, Inc.) and GAPDH served as the internal control. The qPCR conditions were as follows: $95^{\circ} \mathrm{C}$ for $30 \mathrm{sec}, 55^{\circ} \mathrm{C}$ for $30 \mathrm{sec}$, and $72^{\circ} \mathrm{C}$ for $90 \mathrm{sec}$ for 40 cycles, and a final extension at $72^{\circ} \mathrm{C}$ for $10 \mathrm{~min}$. Thus, the analysis of the relative gene expression data was conducted using SYBR ${ }^{\circledR}$-Green Real-time PCR Master Mix (Vazyme Biotech Co., Ltd.) and the $2^{-\Delta \Delta C q}$ method (19). The primers used are listed in Table I.

Plasmid and transfection. The siRNA- negative control (NC, UUCUCCGAACGUGUCACGUAC), siRNA-1 (AGAGUUAC UUUAAUGACUCAG) and siRNA-2 (AAACCAUAGCCUAU GGUUGUC) were constructed by GenePharma. We transfected 
A GSE27155
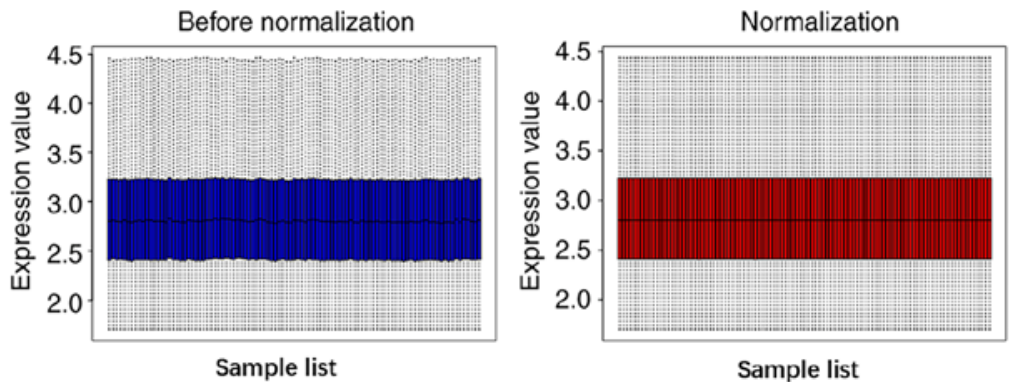

Sample list
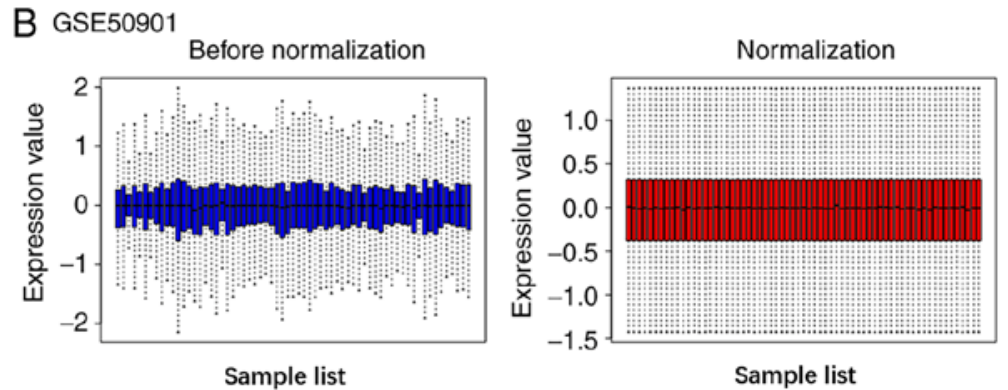
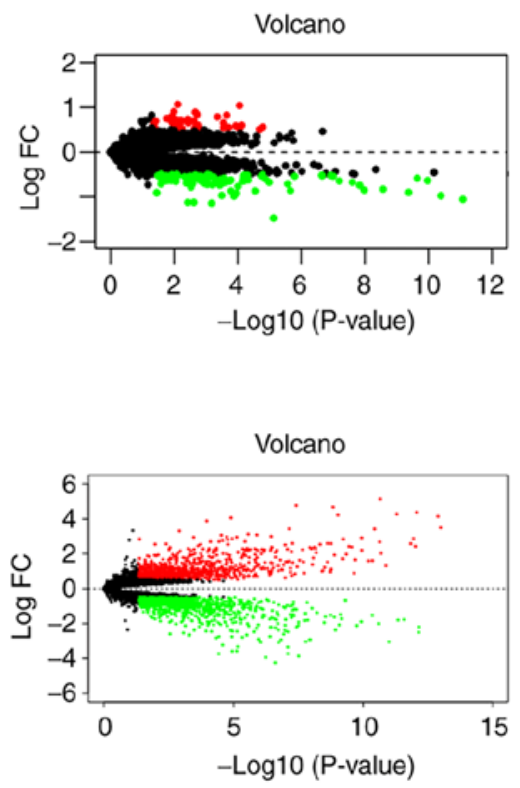

Figure 1. Differential expression of genes in two datasets from the Gene Expression Omnibus database. (A) Standardization (left and middle) and volcano plot (right) of the GSE27155 data. (B) Standardization (left and middle) and volcano plots (right) of the GSE50901 data. The data prior to and after normalization are presented as blue and red bars, respectively. The red and green points respectively represented upregulated and downregulated genes screened based on $\mid \mathrm{FCl}>2.0$ and a corrected $\mathrm{P}<0.05$. The black dots represent genes with no significant difference. FC, fold change.

Table I. Primers used for PCR.

\begin{tabular}{llc}
\hline Gene & Primer direction & Sequence (5' to 3') \\
\hline KCNJ2 & Forward & GTCCCCAACACTCCCCTTTG \\
& Reverse & CAGCTTGCCGTCTCTCATGG \\
GAPDH & Forward & ACACCCACTCCTCCACCTTT \\
& Reverse & TTACTCCTTGGAGGCCATGT \\
\hline
\end{tabular}

KCNJ2, potassium inwardly rectifying channel subfamily J member 2.

the plasmids into CAL-62 and TPC-1 cells with Lipofectamine 2000 Transfection Reagent (Invitrogen; Thermo Fisher Scientific, Inc.) according to the manufacturer's protocol.

Cell Counting Kit-8 (CCK-8) assay. The CCK-8 assay was performed to measure the proliferation of CAL-62 and TPC-1 cells. Cells were seeded in 96-well plates at a density of 104 cells/well and incubated for 1, 2 or 3 days. Subsequently, CCK-8 reagent (Dojindo) was added, followed by incubation for another $2 \mathrm{~h}$. For measuring the optical density at $450 \mathrm{~nm}$, an automated microplate reader (Synergy4; BioTek) was used.

Flow cytometric assay. To evaluate apoptosis levels, CAL-62 and TPC-1 cells were transfected with negative control siRNA (siRNA-NC, UUCUCCGAACGUGUCACGUAC; GenePharma Co., Ltd.) or siRNA-1 (AGAGUUACUUUAAUGACUCAG; GenePharma Co., Ltd.) against KCNJ2 for $24 \mathrm{~h}$ using the Lipofectamine $2000^{\circledR}$ Transfection Reagent (Invitrogen; Thermo Fisher Scientific, Inc.) . The cells were collected and an Annexin V-FITC/PI apoptosis detection kit (Invitrogen; Thermo
Fisher Scientific, Inc.) was used to label the CAL-62 and TPC-1 cells with Annexin V and PI. The cells were then analyzed using flow cytometry (FACScan; BD Biosciences) and their fluorescence of PI and FITC was quantified.

Migration and invasion assay. Transwell chambers without or with Matrigel (Corning, Inc.) were utilized for the migration and invasion assay, respectively. In brief, $2 \times 10^{5}$ cells were seeded into each of the upper chambers, which were precoated with Matrigel for the invasion assay, with $200 \mu \mathrm{l}$ serum-free medium. The bottom chambers were filled with $600 \mu \mathrm{l}$ complete medium. The cells were then cultured at $37^{\circ} \mathrm{C}$ in an atmosphere containing $5 \% \mathrm{CO}_{2}$ according to the manufacturer's protocol. After $24 \mathrm{~h}$, the cells that had migrated or invaded through the filter to the lower chamber were fixed with $4 \%$ paraformaldehyde for $20 \mathrm{~min}$ at room temperature and stained with $1 \%$ crystal violet for $20 \mathrm{~min}$ at room temperature and then counted under a microscope.

Statistical analysis. All tests were performed using GraphPad Prism 6.0 software (GraphPad Software, Inc.). Values are expressed as the mean \pm standard deviation and were analyzed using a paired Student's t-test (parametric) or ANOVA with the Scheffé post-hoc test (for multiple comparisons). $\mathrm{P}<0.05$ was considered to indicate statistical significance.

\section{Results}

Microarray data analysis and identification of DEGs in TC. The mRNA microarray datasets GSE27155 and GSE50901 for TC were downloaded and expression levels were normalized (Fig. 1A and B, left and middle panels). The two datasets were then screened using the limma package [log fold 
A

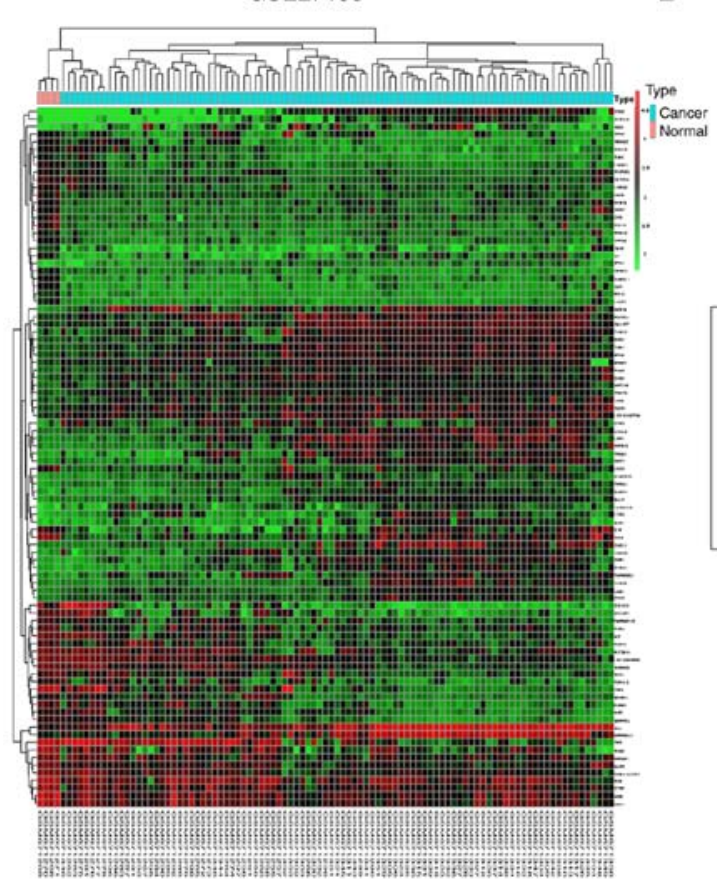

B

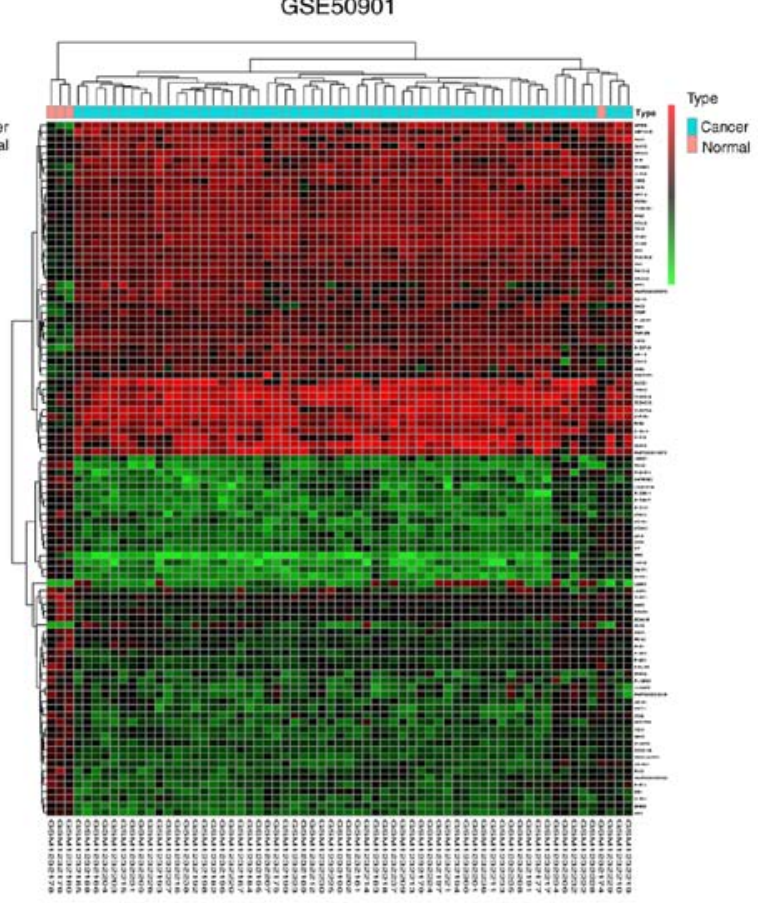

C Up-regulated genes

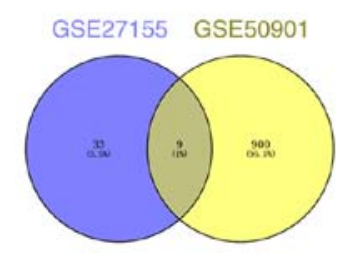

Down-regulated genes

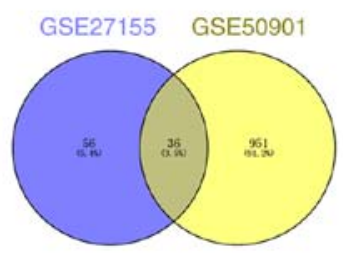

Figure 2. DEG analysis in thyroid carcinoma. (A and B) Heat maps of overlapped DEGs determined from the datasets (A) GSE27155 and (B) GSE53072, respectively. (C) Venn diagram analysis revealed 134 and 1,896 DEGs in the GSE27155 and GSE50901 dataset, respectively, and there were 9 upregulated and 36 downregulated DEGs in common. DEG, differentially expressed gene.

change $($ FC) $>1$ and false discovery rate $<0.05$ ] to obtain DEGs. Volcano plots were used to display the differential expression of various genes from each dataset (Fig. 1A and B, right panel). The heat maps revealed 134 DEGs from the GSE27155 dataset and 1,896 DEGs from the GSE50901 dataset (Fig. 2A and B). A total of 9 upregulated genes and 36 downregulated genes were obtained after integrated analysis, as illustrated in a Venn diagram (Fig. 2C).

Functional enrichment analysis. The DAVID online database was used for biological annotation of the identified common DEGs from the integrated analysis of the two microarray datasets for TC. GO functional enrichment of up- and downregulated genes with a P-value $<0.05$ was performed. The functioal terms of the common DEGs were determined in the categories MF, BP and $\mathrm{CC}$ in the GO analysis (Fig. 3A and B). In the category MF, the common DEGs were mainly enriched in protein binding, oxidoreductase activity and aldehyde dehydrogenase activity. In the category BP, the common DEGs were mainly enriched in cardiac muscle cell potential involved in contraction, platelet degranulation and locomotory exploration behaviors. In the category $\mathrm{CC}$, the selected DEGs were mainly enriched in the plasma membrane, apical plasma membrane and neuronal cell body. The KOBAS online analysis platform was used to analyze the common DEGs identified from the integrated analysis of gene microarrays for TC. The most significantly enriched pathways of DEGs were determined by KEGG analysis. The signaling pathways of DEGs were mainly enriched in the canonical Wnt signaling pathway and the TGF- $\beta$ receptor signaling pathway. These data were subsequently imported into Cytoscape (http://www.cytoscape.org/) to calculate the topological characteristics of the network and to determine each node. $\mid \log \mathrm{FCl}>2$ was individually set as the cutoff criterion to obtain a distinct PPI network for the top 9 


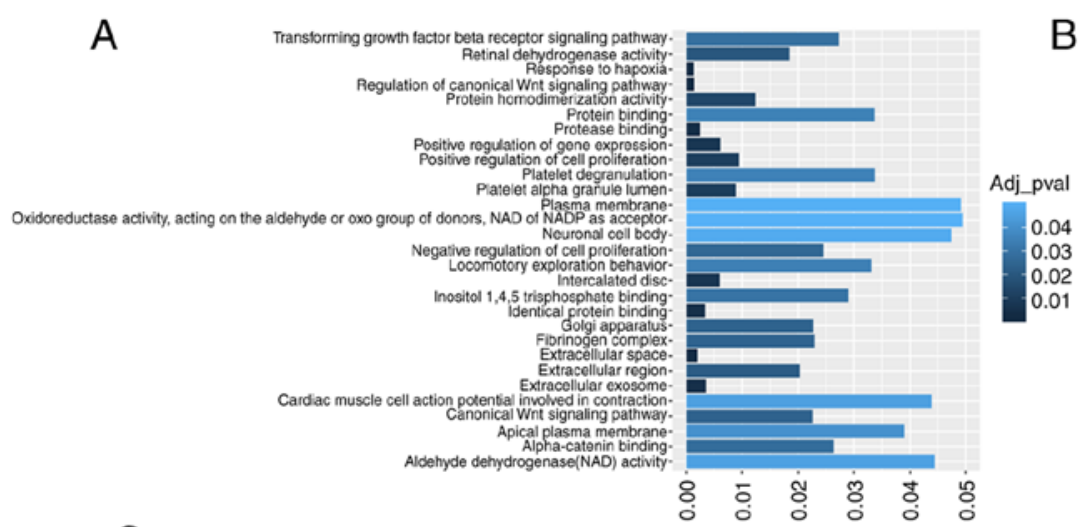

B

C
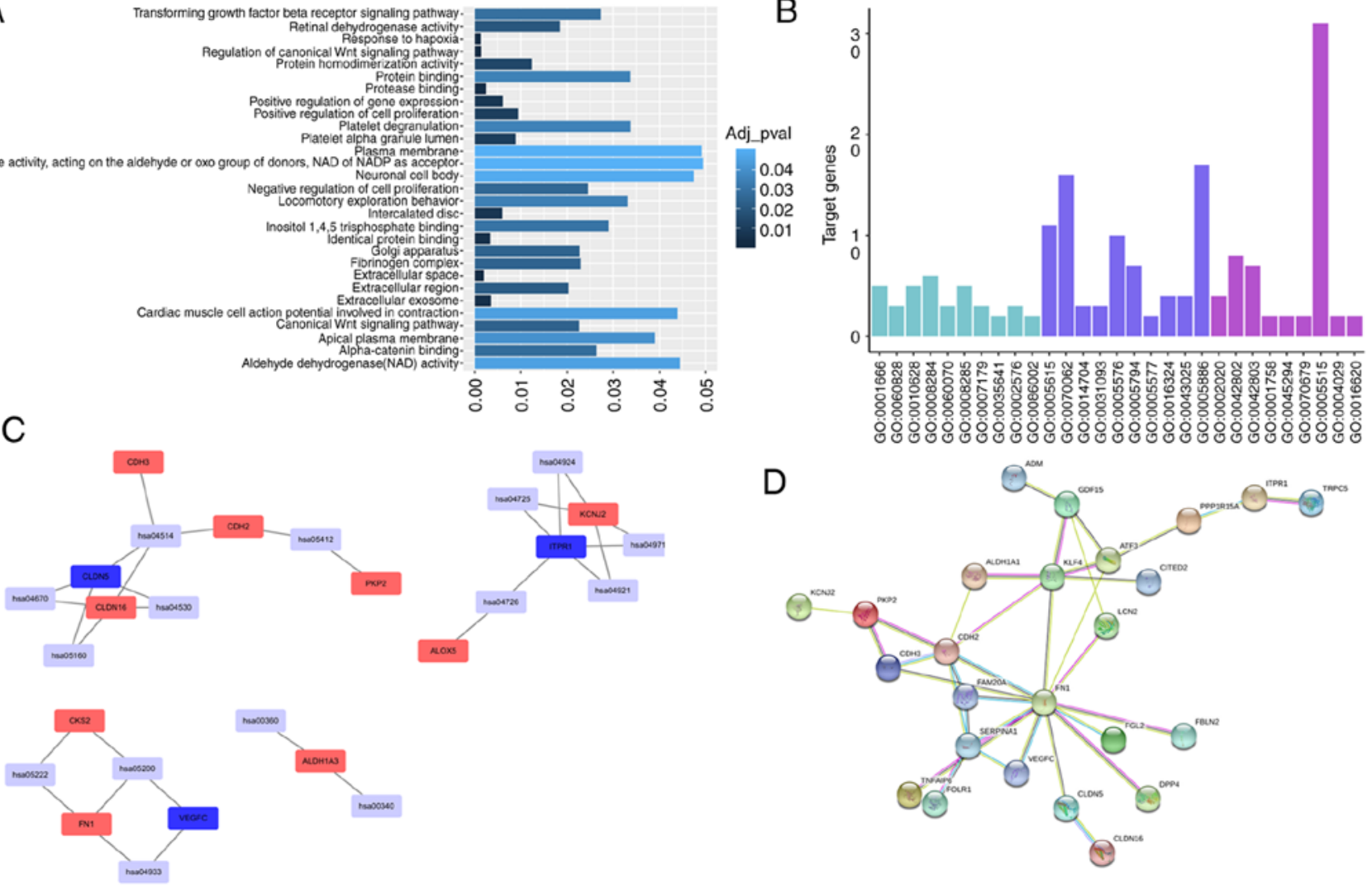

Figure 3. Functional enrichment analysis to provide GO terms and KEGG pathways, and PPI network for common DEGs. (A) The adjusted P-value of GO terms enriched by the DEGs. (B) GO analysis of DEGs with terms divided into three functional groups: Cellular component, molecular function and biological process (from left to right). (C) PPI networks constructed for the common DEGs. Red, blue and light blue represent the upregulated genes, downregulated genes and non-DEGs, respectively. (D) Top 9 up- (red) and downregulated (blue) hub genes in a PPI network. DEG, differentially expressed gene; adj_pval, adjusted P-value; PPI, protein-protein interaction; GO, gene ontology; KEGG, Kyoto Encyclopedia of Genes and Genomes; hsa, Homo sapiens.

common upregulated DEGs (Fig. 3C). Based on the information from the STRING database, the top 9 common up- and downregulated DEGs with higher degrees were selected as hub genes and displayed a strong association with other node proteins (Fig. 3D).

Oncogenic role of $K C N J 2$ in TC. Based on the integrated analysis above, $\mathrm{KCNJ} 2$ was the most significant upregulated gene among the common DEGs (Table SI). The KCNJ2 gene, first cloned from a macrophage cell line in 1993 (20), encodes Kir2.1 and belongs to the classical, inwardly rectifying family of potassium channels (21). To explore the expression of KCNJ2 in TC tissues, RT-qPCR analysis was used to determine the transcriptional levels of $\mathrm{KCNJ} 2$ and the apparent upregulation of $\mathrm{KCNJ} 2$ in $\mathrm{TC}$ tissues compared with the corresponding normal tissues was confirmed (Fig. 4A). The basic information of the cases of TC is provided in Table SII. There were a total of 13 patients $>60$ years and 28 patients $\leq 60$ years. A total of 25 females and 16 males were included in the cuttent study. Furthermore, the impact of KCNJ2 levels on the 5-year survival rate of patients with TC was explored by dividing the patients into two groups with higher or lower KCNJ2 gene expression (the median value was used as the cut off). The results indicated that patients with TC with higher KCNJ2 gene expression had a lower 5-year survival rate compared with that of the patients with lower KCNJ2 gene expression (Fig. 4B), which implies that upregulation of $\mathrm{KCNJ} 2$ may promote $\mathrm{TC}$ progression, leading to reduced survival. In addition, the TC cell lines GLAG-66, CAL-62, B-CPAP, TPC-1 and SW1736 and the human normal thyroid cell line Nthy-ori 3-1 were analyzed. The mRNA levels of KCNJ2 in TC cell lines was significantly higher than that in the control cell line according to RT-qPCR analysis (Fig. 4C). To demonstrate the potential role of $\mathrm{KCNJ} 2$ in cell growth, two siRNAs were constructed to knock down the mRNA level of KCNJ2 in CAL-62 and TPC-1 cells. After transfection of siRNA-1 and -2, downregulation of KCNJ2 was observed in TC cells (Fig. 4D). The CCK-8 assay indicated that the proliferation of TC cells was inhibited in the siRNA-1 treated group as compared with that in the control in two TC cell lines, indicating that KCNJ2 was able to promote the proliferation of TC cells and may have an implication in the tumorigenesis of TC (Fig. 4E). Apoptosis detection indicated that downregulation of $\mathrm{KCNJ} 2$ promoted apoptosis of CAL-62 and TPC-1 cells (Fig. 5A). The migration and invasion assays revealed that decreased expression of KCNJ2 in the siRNA-1 treated groups inhibited cell migration and invasion as compared with that in the controls (Fig. 5B). Taken together, integrated bioinformatics analysis was used to identify the KCNJ2 gene as the most significantly upregulated DEG in TC cells. In addition, it was observed that inhibition of $\mathrm{KCNJ} 2$ reduced the malignant behaviour of TC cells, an indication that $\mathrm{KCNJ} 2$ may be a promising potential diagnostic and prognostic biomarker, and may be a therapeutic target for TC. 
A
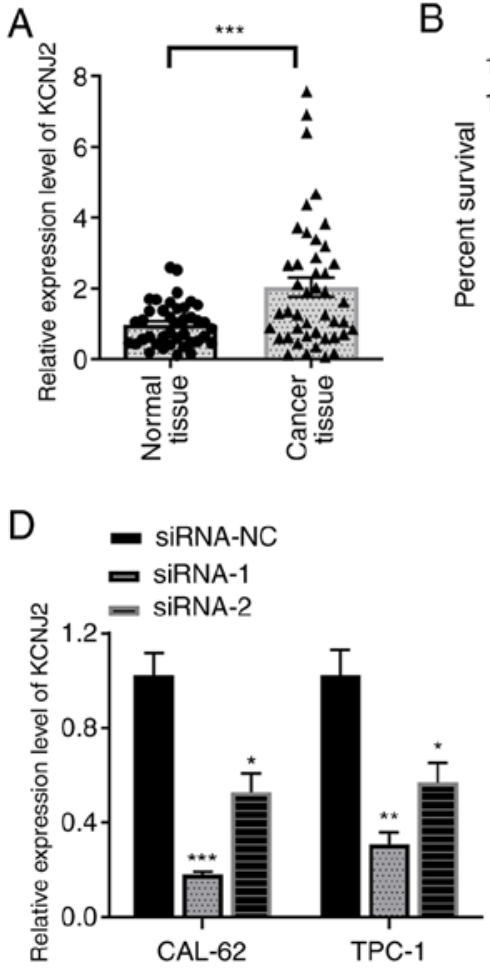

B

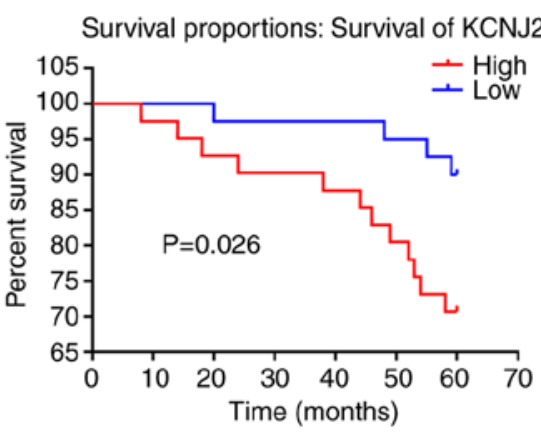

E

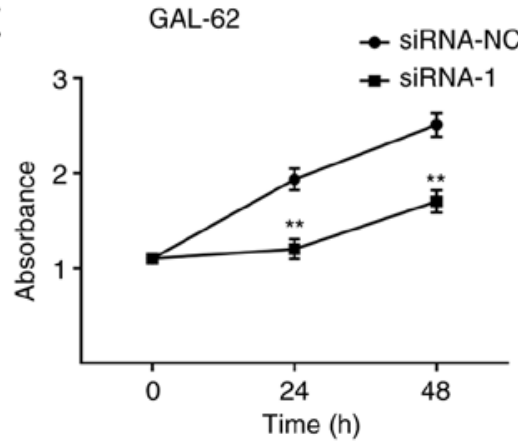

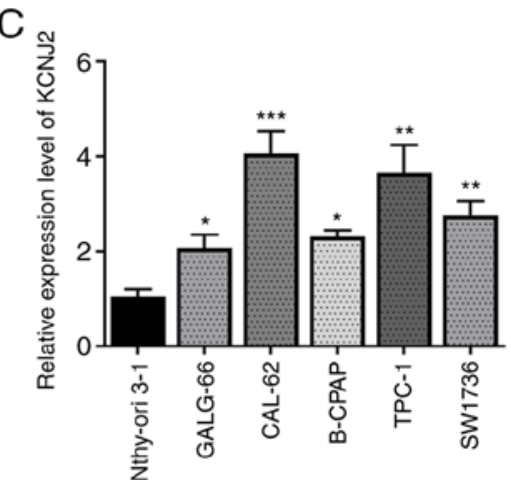

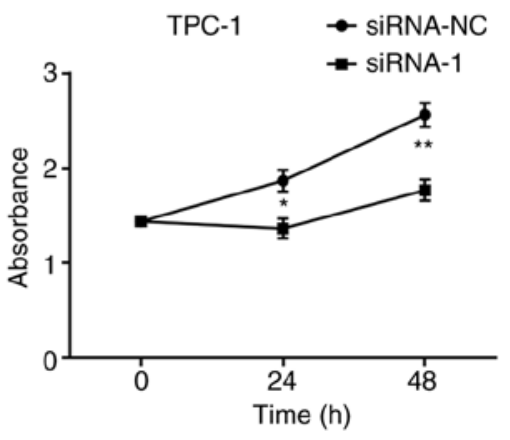

Figure 4. Upregulation of KCNJ2 facilitates malignant behaviours associated with cancer progression in TC cells. (A) Transcriptional levels of KCNJ2 in TC and normal tissues. (B) 5-year survival rate of patients with TC stratified based on higher and lower KCNJ2 expression (P=0.026). (C) mRNA levels of KCNJ2 in TC and normal cell lines. (D) The expression of KCNJ2 was downregulated after transfection of siRNA-1 and -2 into CAL-62 and TPC-1 cells. (E) The cell proliferation assay indicated that knockdown of KCNJ2 reduced the growth of CAL-62 and TPC-1 cells. The values were normalized to the control group. ${ }^{*} \mathrm{P}<0.05,{ }^{* *} \mathrm{P}<0.01$ and ${ }^{* * *} \mathrm{P}<0.001$ vs. Nthy-ori 3-1 in C or vs. siRNA-NC in D and E. KCNJ2, potassium inwardly rectifying channel subfamily $\mathrm{J}$ member 2 ; TC, thyroid cancer; siRNA, small interfering RNA; NC, negative control.

\section{Discussion}

TC is the most common endocrine malignancy, accounting for $0.5-1 \%$ of all human cancers, and ranks fifth among the cancer types in females (1-5). Despite advanced surgical techniques and medical treatments for TC, the survival rate of affected patients remains low $(7,10)$. Thus, early diagnosis of TC is important. However, diagnostic and prognostic biomarkers for TC are currently lacking. In the past decade, microarray technology has been widely used in numerous studies to profile gene expression and to examine DEGs and their distinct pathways, as well as GO functions in the categories BP, MF and CC (12-14). Several studies have previously studied thyroid gene expression profiles and screened thousands of DEGs, which may be involved in the progression of TC (5,7-9). Using integrated bioinformatics analyses of microarray data from GEO, investigators have revealed key genes and pathways associated with ATC or PTC $(5,8)$. However, biomarkers for diagnostic and prognostic applications of TC still deserve further exploration.

Using integrated bioinformatics analysis, the present study aimed to discover key genes and pathways involved in TC. Human thyroid adenomas, carcinomas and papillary thyroid carcinomas are all types of $\mathrm{TC}$ and the present analysis focused on the difference in expression patterns between normal tissues and TC tissues. The GSE27155 and GSE50901 datasets, which include different subtypes of TC, were selected for the present analysis. To obtain the DEGs, the mRNA profiles of GSE27155 and GSE50901 were downloaded from the GEO database and 134 and 1,896 DEGs were respectively identified from them. Of note, the expression levels in the two datasets exhibited certain differences. A possible reason why the expression values or the number of DEGs were lower in the GSE27155 dataset compared with those in the GSE50901 dataset was that the patients included in the two datasets were from different countries (Brazil and USA, respectively). Among them, 9 upregulated and 36 downregulated common DEGs were identified. Subsequently, GO term and KEGG pathway enrichment analyses were performed to determine the biological functions of the DEGs and it was identified that the common DEGs were primarily enriched in protein binding, cardiac muscle cell potential involved in contraction, aldehyde dehydrogenase activity, TGF- $\beta$ receptor signaling pathway and the canonical Wnt signaling pathway. In addition, a PPI network was constructed to indicate the interactions of the top 9 up- and downregulated DEGs, such as KCNJ2, cadherin 3, Cbp/p300 interacting transactivator with Glu/Asp rich carboxy-terminal domain 2 and VEGFC.

Kir2.1 is encoded by the KCNJ2 gene and belongs to the classical inwardly rectifying family of potassium channels (21). Previous studies proved that Kir2.1 has a strong inward rectifier $\mathrm{K}^{+}$current in multiple cell types, including neurons, skeletal muscle, cardiac myocytes and carcinoma cells $(22,23)$. In addition, the potential roles of the $\mathrm{KCNJ} 2$ gene in TC cells were further explored. The upregulation of KCNJ2 in TC tissues and cell lines was confirmed using RT-qPCR. Furthermore, the expression levels of KCNJ were determined to be significantly associated with the 5-year survival rate of patients with TC. 
A

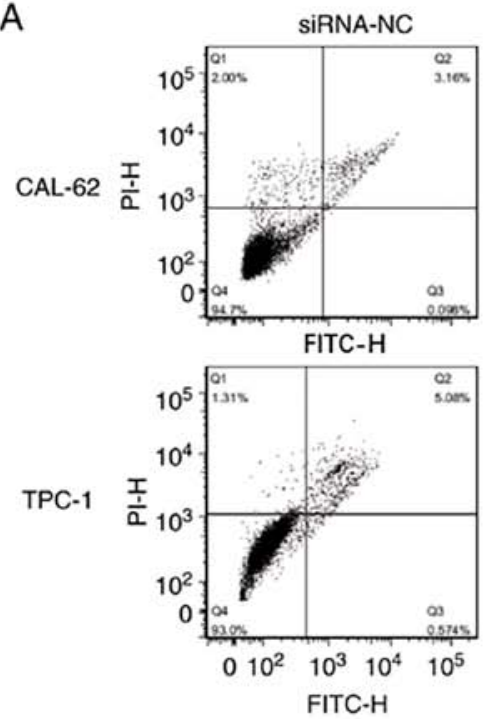

B
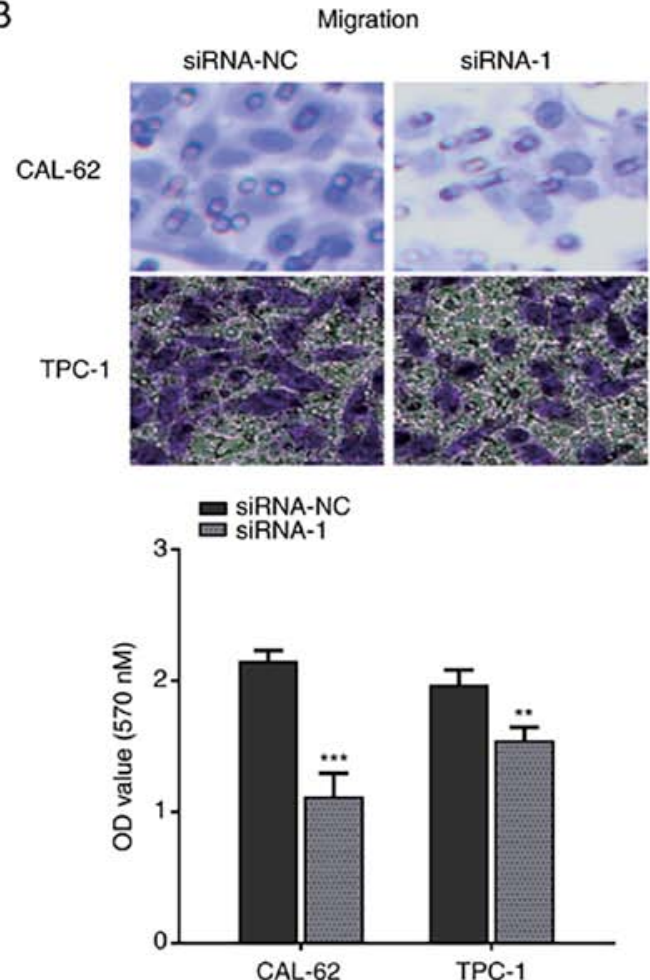

siRNA-1
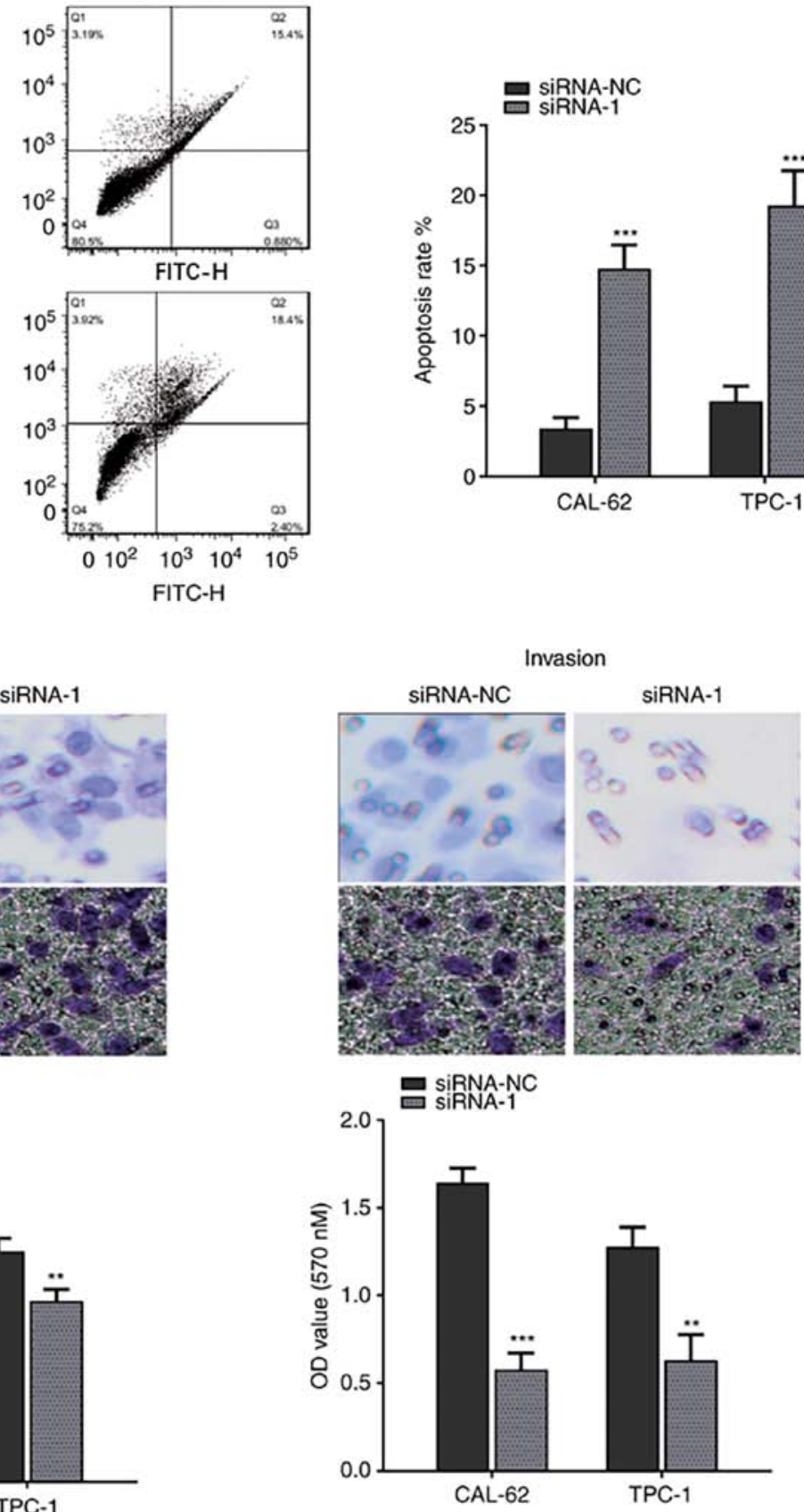

Figure 5. Knockdown of KCNJ2 inhibits cell apoptosis while facilitating migration and invasion of thyroid cancer cells. (A) A cell apoptosis assay demonstrated that knockdown of KCNJ2 expression upregulated the apoptosis rate of CAL-62 and TPC-1 cells. (B) Transwell assays (magnification, x100) indicated that decreased KCNJ2 expression inhibited the migration and invasion ability of CAL-62 and TPC-1 cells, which are processes associated with the ability of cancer to metastasize. ${ }^{* *} \mathrm{P}<0.01$ and ${ }^{* * *} \mathrm{P}<0.001$ vs. siRNA-NC. Q, quadrant; OD, optical density; KCNJ2, potassium inwardly rectifying channel subfamily $\mathrm{J}$ member 2; siRNA, small interfering RNA; NC, negative control.

Finally, it was revealed that knockdown of $\mathrm{KCNJ} 2$ repressed the growth and metastasis, while increasing the apoptosis rate of TC cells, suggesting an oncogenic role of KCNJ2 during TC development and progression. A previous study by Liu et al (21) elucidated the role of KCNJ in small-cell lung cancer (SCLC) and indicated that overexpression of KCNJ promoted cell growth and inhibited apoptosis. Furthermore, they established the association between $\mathrm{KCNJ} 2$ and multidrug resistance protein 1/ATP binding cassette subfamily $\mathrm{C}$ member 1 . In addition, they reported that upstream microRNA-7 and the Ras/MAPK pathway could regulate the expression of KCNJ2 during SCLC development (21). Further investigation is required on whether the $\mathrm{KCNJ} 2$ has a similar role in TC.

In conclusion, integrated bioinformatics analysis was used to identify 45 common DEGs in two GEO datasets. Among the most significantly upregulated genes, $\mathrm{KCNJ} 2$ was indicated to have an important role in regulating malignant behaviours of TC cells that may be involved in tumorigenesis and progression, which strongly suggests that KCNJ2 may be a promising biomarker for diagnosis, prognosis and prevention of TC. Due to its oncogenic role, it may also be a therapeutic target for patients with TC. 


\section{Acknowledgements}

Not applicable.

\section{Funding}

The present study was supported by funding for key disciplines of Inner Mongolia Autonomous Region (grant no. pwk 03).

\section{Availability of data and materials}

All data generated or analyzed during this study are included in this published article.

\section{Authors' contributions}

XH designed the study. BZ and ZC performed RT-qPCR analysis, the CCK-8 assay and plasmid transfection. YW conducted the other functional experiments. GF collected and analyzed the data. ZC performed the statistical analysis, performed a literature search and contributed to manuscript editing. BZ wrote the manuscript. All authors have read and approved the final manuscript. BZ and $\mathrm{ZC}$ confirm the authenticity of all the raw data.

\section{Ethics approval and consent to participate}

Written informed consent was obtained from each patient and the experimental procedures were all in accordance with the guidelines of the Ethics Committee of Inner Mongolia Autonomous Region People's Hospital (Hohhot, China). The present study was approved by the Ethics Committee of Inner Mongolia Autonomous Region People's Hospital (Hohhot, China; no. 202000406L).

\section{Patient consent for publication}

Not applicable.

\section{Competing interests}

The authors declare they have no competing interests.

\section{References}

1. Siegel RL, Miller KD and Jemal A: Cancer statistics, 2019. CA Cancer J Clin 69: 7-34, 2019.

2. Pacini F: Observation for newly diagnosed micro-papillary thyroid cancer: Is now the time? J Endocrinol Invest 38: 101-102, 2015.

3. Chen W, Zheng R, Baade PD, Zhang S, Zeng H, Bray F, Jemal A, Yu XQ and He J: Cancer statistics in China, 2015. CA Cancer J Clin 66: 115-132, 2016.

4. Lodewijk L, Prins AM, Kist JW, Valk GD, Kranenburg O, Rinkes IH and Vriens MR: The value of miRNA in diagnosing thyroid cancer: A systematic review. Cancer Biomark 11: 229-238, 2012.

5. Yu J, Mai W, Cui Y and Kong L: Key genes and pathways predicted in papillary thyroid carcinoma based on bioinformatics analysis. J Endocrinol Invest 39: 1285-1293, 2016.
6. Smallridge RC, Marlow LA and Copland JA: Anaplastic thyroid cancer: Molecular pathogenesis and emerging therapies. Endocr Relat Cancer 16: 17-44, 2009.

7. Geraldo MV and Kimura ET: Integrated analysis of thyroid cancer public datasets reveals role of post-transcriptional regulation on tumor progression by targeting of immune system mediators. PLoS One 10: e0141726, 2015.

8. Hu S, Liao Y and Chen L: Identification of key pathways and genes in anaplastic thyroid carcinoma via integrated bioinformatics analysis. Med Sci Monit 24: 6438-6448, 2018.

9. Fluge $\varnothing$, Bruland O, Akslen LA, Lillehaug JR and Varhaug JE: Gene expression in poorly differentiated papillary thyroid carcinomas. Thyroid 16: 161-175, 2006.

10. Garg M, Kanojia D, Okamoto R, Jain S, Madan V, Chien W, Sampath , Ding LW, Xuan M, Said JW, et al: Laminin-5 $\gamma-2$ (LAMC2) is highly expressed in anaplastic thyroid carcinoma and is associated with tumor progression, migration, and invasion by modulating signaling of EGFR. J Clin Endocrinol Metab 99: E62-E72, 2014.

11. Kulasingam V and Diamandis EP: Strategies for discovering novel cancer biomarkers through utilization of emerging technologies. Nat Clin Pract Oncol 5: 588-599, 2008.

12. Liu YJ, Zhang S, Hou K, Li YT, Liu Z, Ren HL, Luo D and Li SH: Analysis of key genes and pathways associated with colorectal cancer with microarray technology. Asian Pac J Cancer Prev 14: 1819-1823, 2013.

13. Wang J, Wei B, Cao S, Xu F, Chen W, Lin H, Du C and Sun Z: Identification by microarray technology of key genes involved in the progression of carotid atherosclerotic plaque. Genes Genet Syst 89: 253-258, 2014.

14. Wang Y and Zheng T: Screening of hub genes and pathways in colorectal cancer with microarray technology. Pathol Oncol Res 20: 611-618, 2014.

15. Goujon M, McWilliam H, Li W, Valentin F, Squizzato S, Paern J and Lopez R: A new bioinformatics analysis tools framework at EMBL-EBI. Nucleic Acids Res 38: W695-9, 2010.

16. Rhodes DR, Yu J, Shanker K, Deshpande N, Varambally R, Ghosh D, Barrette T, Pandey A and Chinnaiyan AM: ONCOMINE: A cancer microarray database and integrated data-mining platform. Neoplasia 6: 1-6, 2004.

17. Giordano TJ, Kuick R, Thomas DG, Misek DE, Vinco M, Sanders D, Zhu Z, Ciampi R, Roh M, Shedden K, et al: Molecular classification of papillary thyroid carcinoma: Distinct BRAF, RAS, and RET/PTC mutation-specific gene expression profiles discovered by DNA microarray analysis. Oncogene 24 : 6646-6656, 2005.

18. Barros-Filho MC, Marchi FA, Pinto CA, Rogatto SR and Kowalski LP: High diagnostic accuracy based on CLDN10, HMGA2, and LAMB3 transcripts in papillary thyroid carcinoma. J Clin Endocrinol Metab 100: E890-E899, 2015.

19. Kenneth L and Thomas S: Analysis of relative gene expression data using real-time quantitative PCR and the $2^{-\Delta \Delta C T}$ method. Methods 25: 402-408, 2005.

20. Kubo Y, Baldwin TJ, Jan YN and Jan LY: Primary structure and functional expression of a mouse inward rectifier potassium channel. Nature 362: 127-133, 1993.

21. Liu H, Huang J, Peng J, Wu X, Zhang Y, Zhu W and Guo L: Upregulation of the inwardly rectifying potassium channel Kir2.1 (KCNJ2) modulates multidrug resistance of small-cell lung cancer under the regulation of miR-7 and the Ras/MAPK pathway. Mol Cancer 14: 59, 2015.

22. Li M, Kanda Y, Ashihara T, Sasano T, Nakai Y, Kodama M, HayashiE, Sekino Y,Furukawa T and Kurokawa J: Overexpression of KCNJ2 in induced pluripotent stem cell-derived cardiomyocytes for the assessment of QT-prolonging drugs. J Pharmacol Sci 134: 75-85, 2017.

23. Giovannardi S, Forlani G, Balestrini M, Bossi E, Tonini R, Sturani E, Peres A and Zippel R: Modulation of the inward rectifier potassium channel IRK1 by the Ras signaling pathway. J Biol Chem 277: 12158-12163, 2002. 